\title{
Equipe de enfermagem $x$ acompanhante na pediatria: o impacto dessa parceria na assistencia pediatrica
}

RESUMO |Este estudo trata-se de uma pesquisa descritiva, exploratória e de cunho qualitativo. Mas, que não exclui a presença de dados quantitativos, na medida em que inclui tabelas obtidas através da coleta de dados obtida utilizando-se como instrumento a entrevista semiestruturada, realizada no Hospital da Posse, em Nova Iguaçu - RJ. As perguntas elaboradas e aplicadas tiveram como objetivo a análise da fala dos profissionais de enfermagem na clínica pediátrica, buscando-se entender como a interação entre família e os profissionais enfermeiros no compartilhamento do cuidado à criança hospitalizada e pode representar uma conexão importante para o êxito do tratamento. Pode-se concluir que é importante promover melhorias no processo de comunicação e nas interações que são desenvolvidas, no sentido de considerar o acompanhante como parte fundamental dos cuidados. Da mesma forma, verificou-se a necessidade de utilizar estratégias de humanização hospitalar no atendimento à família e à criança hospitalizada, considerando suas demandas e promovendo a satisfação daqueles que estão envolvidos.

Palavras-chaves: Enfermagem Pediátrica; Família; Criança Hospitalizada.

ABSTRACT | This study is a descriptive, exploratory and qualitative research. However, it does not exclude the presence of quantitative data, as it includes tables obtained through the collection of data obtained using the semi-structured interview conducted at the Posse Hospital in Nova Iguaçu - RJ. The questions elaborated and applied had as objective the analysis of the speech of the nursing professionals in the pediatric clinic, trying to understand how the interaction between family and the professional nurses in the sharing of the care to the hospitalized child and can represent an important connection for the success of the patient. treatment. It can be concluded that it is important to promote improvements in the communication process and the interactions that are developed, in order to consider the companion as a fundamental part of care. Similarly, there was a need to use hospital humanization strategies to care for the family and hospitalized children, considering their demands and promoting the satisfaction of those involved.

Keywords: Pediatric Nursing; Family; Hospitalized Child.

RESUMEN | Este estudio es una investigación descriptiva, exploratoria y cualitativa. Sin embargo, no excluye la presencia de datos cuantitativos, ya que incluye tablas obtenidas a través de la recopilación de datos obtenidos mediante la entrevista semiestructurada realizada en el Hospital Posse en Nova Iguaçu - RJ. Las preguntas elaboradas y aplicadas tuvieron como objetivo el análisis del discurso de los profesionales de enfermería en la clínica pediátrica, tratando de entender cómo la interacción entre la familia y las enfermeras profesionales en el intercambio de la atención al niño hospitalizado y puede representar una conexión importante para el éxito del paciente. tratamientoSe puede concluir que es importante promover mejoras en el proceso de comunicación y las interacciones que se desarrollan, para considerar al acompañante como una parte fundamental de la atención. Del mismo modo, era necesario utilizar estrategias de humanización en el hospital para cuidar a la familia y a los niños hospitalizados, teniendo en cuenta sus demandas y promoviendo la satisfacción de los involucrados.

Descriptores: Enfermería Pediátrica; Familia; Niño Hospitalizado.

\section{Bruna Porath Azevedo Fassarella}

Enfermeira, docente do curso de enfermagem da universidade iguacu(Unig); preceptora acadêmica da universidade Uniabeu; pós graduada em educação em saúde para preceptores do Sus, mestranda em urgência e emergência pela universidade de vassouras.

\footnotetext{
Wanderson Alves Ribeiro

Enfermeiro. Mestre em Ciências do Cuidado em Saúde pela Universidade Federal Fluminense. Docente do Curso de Pós-Gaduação de Enfermagem em Emergência e Terapia Intensiva da UNIG. Rio de Janeiro, RJ.
}

\section{Letícia De Moraes Freitas}

Enfermeira, graduada pela Universidade Iguaçu (UNIG).

\section{Jenifer Cruz Do Nascimento}

Enfermeira, graduada pela Universidade Iguaçu (UNIG).

\section{José Carlos Cezar dos Santos \\ Bacharel em Enfermagem pela Associação Brasileira de Ensino Universitário, UNIABEU, Belford Roxo, Rio de Janeiro.}

\begin{abstract}
Caroliny dos Santos Guimarães da Fonseca
Enfermeira. Mestrado em Bioética e Ética Médica da Universidad Européa Del Atlántico, Espanha. Especialização em Gestão em Enfermagem, Urgência e Emergência e Auditoria em Sistemas de Saúde. Diretora da Global Academic Support. Docente do Curso de Pós-Graduação em Alta Complexidade da Universidade Veiga de Almeida (UVA). Rio de Janeiro, RJ.
\end{abstract}

Recebido em: 22/05/2019

Aprovado em: 25/05/2019 
INTRODUÇÃO

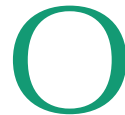

processo de hospitalização é frequentemente marcado por uma combinação de anseios e medos, sensação de insegurança e desconforto para a criança e para a família, em especial para os pais, a mudança na rotina gera desconfiança e alto nível de estresse, além do pior entre todos estes sentimentos: o medo de perder sua criança tão amada para uma enfermidade qualquer ${ }^{(1)}$.

Compreender as repercussões da hospitalização para a criança e sua família, como mudanças na rotina familiar, ausência da mãe em casa, conflitos conjugais e tensão em relação à criança doente é uma habilidade a ser desenvolvida pela equipe de saúde, sendo capaz de fornecer cuidados adequados às necessidades de cada família ${ }^{(2)}$.

Neste contexto, buscar interagir com a família supõe uma atitude ética, qualificada e humanizada, procurando estabelecer um espaço de diálogo que envolva angústias, as expectativas e outros sentimentos dos pais durante o atendimento à sua criança doente no ambiente hospitalar, assim como o compartilhamento das estratégias de tratamento a serem utilizadas, envolvendo-os nos cuidados não como espectadores, mas como verdadeiros partícipes da assistência ${ }^{(3)}$.

No Brasil, a presença dos pais ou responsáveis pela criança dentro do hospital adveio a ser ratificada legalmente através do Art. 12 da Lei n. ${ }^{\circ}$ 8.069, de 1990 - Estatuto da Criança e de Adolescente (ECA). Esta lei distingue e estima a importância da permanência e da participação dos pais no procedimento de recuperação da saúde dos seus filhos e sugere a tentativa de adaptar a unidade quanto à infraestrutura e capacitar os profissionais de saúde para proporcionarem uma assistência apropriada a todos os envolvidos neste processo ${ }^{(4)}$.

A organização do processo de trabaIho supõe a existência de normas e rotinas que possibilitem a convivência pacífica de diferentes pacientes, familiares e profissionais neste contexto, objetivando o cuida- do da criança com qualidade e harmonia, preferencialmente, sem resistências e conflitos, o que só vem a contribuir para o alívio do sofrimento do paciente pediátrico e o restabelecimento pleno da saúde e do bem estar do mesmo ${ }^{(5)}$.

Sob a ótica da Política Nacional de Humanização (PNH), humanizar significa inicialmente valorizar os sujeitos envolvidos (criança, família, equipe de saúde) e, por meio do acolhimento, enfatizar a escuta atenta, desenvolver a capacidade de empatia, oferecer apoio, identificar as queixas, e estabelecer vínculos ${ }^{(6)}$.

A relação entre quem presta e quem recebe o cuidado não adquire sentido e não pode ser vivida, a não ser que: permita uma interação saudável com a pessoa tratada e os que o cercam; estimule a participação ativa; e contribua para assegurar a continuidade do cuidado. Não envolver a família no planejamento, intervenção, tomadas de decisões e evolução não é caracterizado como cuidado centrado na família, portanto, ainda são necessárias políticas e/ ou programas que incluam a família no cenário hospitalar pediátrico ${ }^{(7)}$.

Outro aspecto necessário do cuidado é a preparação da família para a continuidade dos cuidados no domicílio. Apoiar esse processo a partir da alta hospitalar corresponde a uma das dimensões da assistência do enfermeiro. A capacitação do familiar para realizar os cuidados à criança proporciona a continuidade destes no domicílio ${ }^{(8)}$.

Os paradigmas aos quais este estudo se refere nasceram da evolução técnico-científica que acompanhou o surgimento do sistema capitalista. Este modelo, com fortes influências cartesianas, não considerava o homem nas suas múltiplas dimensões, mas restringia-se aos elementos doença-saber/médico-cura. O paciente era um objeto e não um sujeito sob o olhar dos profissionais de saúde, descartado, desvalorizado e sem $\mathrm{voz}^{(9)}$.

O pensar a saúde, com a evolução dos tempos, está deixando de se orientar da forma simplista de antes, como a mera ausência de doença, adotando novas dimensões: o bem-estar físico, psíquico, socioeconômico. E no cerne de tudo, o ser: a pessoa dentro destas dimensões e como parte de uma perspectiva de cuidado muito mais ampla ${ }^{(9)}$.

É incontestável que o paciente precisa de assistência, de estímulo e de um suporte eficiente por parte de uma equipe multi e interdisciplinar, mormente enfermeiro, por ser aquela que mantém durante todo o processo de tratamento até seu termo, uma maior proximidade com o mesmo, relacionada ao cuidar. Mas não há como manter a família apartada de uma situação que envolve a saúde de um ente querido, mormente quando se trata de uma criança ${ }^{(10)}$.

A justificativa deste estudo situa-se em uma reflexão sobre a importância da interação do enfermeiro com os familiares acompanhantes da criança hospitalizada. É uma situação desconfortável assistir sua criança ser manipulada por estranhos, passando por procedimentos, muitas vezes dolorosos; tornando determinante se a equipe age unilateralmente ou se demonstra interesse numa salutar troca de ideias, já que a criança é um ser com muitas singularidades, já bastante conhecidas pela família, mas não pelos profissionais ${ }^{(11)}$.

A essência da assistência de enfermagem deve estar centrada no cuidar integral, que envolva a restauração da saúde e da qualidade de vida. Neste sentido, a contribuição do enfermeiro ultrapassa a meta assistência técnico- científica ao aceitar a família como um grupo social dinâmico e que sua relação com o paciente envolve afetos, simbolismos próprios, códigos de comportamento e que o ato de criar uma conexão entre enfermagem-criança-família pode ser gratificante para todos ${ }^{(12)}$.

Pensar a família como contexto e foco de cuidado é um desafio que envolve ir além de orientar e esperar a colaboração no desempenho de ações de cuidado em relação ao paciente que demanda atenção. Afinal, o acompa- 
nhante deve ser, não apenas na teoria, mas na prática dos serviços de saúde, um parceiro da enfermagem, respeitado e essencial no cuidar humanizado ao paciente ${ }^{(13)}$.

O cuidado destinado à criança e à família torna-se fragmentado quando se considera exclusivamente os princípios institucionais do ambiente hospitalar. Assim, a valorização das vivências da criança e de sua família representa o principal aspecto a ser contemplado pela equipe de enfermagem num cuidar que se pretenda humanizado(6).

Ao adentrar o ambiente hospitalar com todo o seu pragmatismo, sua cientificidade, sua dinâmica rigorosa de procedimentos médicos e de enfermagem, as famílias veem-se submetidas a normas e rotinas impostas pela instituição como forma de organizar o processo de trabaIho e harmonizar as funções dos diversos setores que coabitam aquele ambiente. Fica evidente que essas características ambientais se apresentam como mecanismos de poder disciplinar, com vistas a padronizar a atuação da família no interior da instituição ${ }^{(5)}$.

Ao longo da internação hospitalar da criança, é extremamente relevante que sua família se reconheça como sujeito de direitos, exercendo sua autonomia frente à equipe de saúde e auxiliando na busca, solicitação e luta pelos direitos dos seus familiares internados. Nesse contexto, para que cumpram objetivos, normas e rotinas no hospital, deve-se sempre levar em consideração as necessidades da criança e seus familiares cuidadores na unidade, qualificando seu cuidado, pois, assim, tornam-se parceiros importantes da equipe de enfermagem.

A questão norteadora deste estudo é a seguinte: Qual a percepção do enfer- meiro em relação ao acompanhante da criança hospitalizada? Diante ao exposto, objetivou-se compreender como o enfermeiro e as famílias compartilham o cuidado à criança hospitalizada e como esta conexão é importante para o êxito do tratamento

\section{METODOLOGIA}

Trata-se de uma pesquisa com olhar descritivo e exploratório de cunho qualitativo, onde se optou pela aplicação de entrevista semiestruturada a ser realizada no Hospital de Nova Iguaçu (HGNI), cujo objetivo foi entender como o enfermeiro e as famílias compartilham o cuidado à criança hospitalizada e como esta conexão é importante para o êxito do tratamento. Após a realização da coleta de dados, os quais foram quantificados e organizados em tabelas.

Pelo fato de envolver pessoas na pesquisa de campo, o estudo foi submetido ao Comitê de Ética em Pesquisa da Faculdade de Ciências Biológicas e da Saúde da Universidade Iguaçu (CEP/UNIG), com parecer positivo n. ${ }^{\circ}$ 2.959.325; atendendo à Resolução n. ${ }^{\circ}$ 466/12 do Conselho Nacional de Saúde (CNS), que disserta sobre pesquisa com seres humanos.

A pesquisa foi realizada em 2018 utilizando-se a técnica da entrevista estruturada aplicada aos familiares acompanhantes de crianças hospitalizadas na clínica pediátrica individualmente, desde que aceitaram (por consentimento escrito) participar da pesquisa. As pesquisadoras garantiram o anonimato dos entrevistados. Para tanto, os mesmos foram identificados pela letra E (entrevistados), seguido do número de ordem em que foram responderam às entrevistas. Além disso, ficou bem esclarecido que não existia obrigatoriedade em participar e a recusa não lhes causaria prejuízos de qualquer natureza. O objetivo era atingir 20 enfermeiros que se disponibilizassem a responder às perguntas.

Todas as entrevistas foram gravadas e transcritas a fim de garantir a transparência dos dados obtidos. O tratamento dado às informações coletadas através das entrevistas consistiu numa leitura atenta, buscando agrupar as respostas conforme o grau de incidência a fim de sistematizá-las em tabelas que foram posteriormente elaboradas. Metodologicamente, a pesquisa foi subsidiada pela Análise de Conteúdo desenvolvida por Bardin ${ }^{(14)}$.

A análise de conteúdo, segundo Bardin(14), deve obedecer algumas fases: pré-análise, que compreende a leitura flutuante do material coletado; exploração do material, que consiste na construção das operações de codificação, na definição de regras de contagem, classificação e agregação das informações em categorias simbólicas ou temáticas e, por fim, o tratamento dos resultados, inferência e interpretação.

\section{RESULTADOS E DISCUSSÃO}

Foram analisados os dados e discutidos os resultados obtidos na pesquisa de campo, tendo como finalidade oferecer respostas à questão norteadora proposta no projeto anteriormente apresentado: Qual a percepção do familiar acompanhante da criança frente ao cuidado humanizado?

Os participantes foram identificados pela letra "F" e números, seguindo a ordem de realização das entrevistas.

Quadro 1. Classificação dos sujeitos do estudo em relação ao gênero. Rio de Janeiro, RJ, Brasil, 2018

$\begin{array}{lcc}\text { Sexo } & \text { QUANTIDADE } & \text { PORCENTAGEM } \\ \text { Feminino } & 15 & 75 \% \\ \text { Masculino } & 5 & 25 \% \\ \text { Total } & 20 & 100 \%\end{array}$


Os dados coletados acima corroboram a ideia de estudo ${ }^{(15)}$, que diz que as atividades de cuidar sejam no âmbi- to familiar ou no âmbito institucional são desenvolvidas majoritariamente por mulheres, pois historicamente se
Ihes atribui certas qualidades inatas em oposição às qualificações aprendidas no espaço público.

\section{Quadro 2. Classificação etária dos sujeitos do estudo. Rio de Janeiro, RJ, Brasil, 2018}

\section{Idade}

Abaixo de 30 anos

Entre 31 e 40 anos

Entre 41 e 50 anos

Entre 51 e 60 anos

Acima de 61 anos

Total

De acordo com o que se pode observar no Quadro 2, fracionado em cinco faixas etárias variáveis entre indivíduos menores de 30 anos até indivíduos com mais de 61 anos, depreende-se que
QUANTIDADE

7 6

6

1

20

\section{PORCENTAGEM}

$35 \%$

$30 \%$

$30 \%$

$5 \%$

$0 \%$

$100 \%$

\section{Quadro 3. Temo de serviço em Pediatria dos sujeitos do estudo. Rio de Janeiro, RJ, Brasil, 2018}

\section{Tempo de Serviço em Pediatria}

Abaixo de 1 ano

Entre 1 e 5 anos

Entre 5 e 10 anos

Entre 10 e 15 anos

Acima de 20 anos

Total

Conforme o que se pode observar no Quadro 3, igualmente fracionado em cinco faixas, variáveis entre menos de 1 ano de atividade profissional na área de pediatria até mais de 20 anos na referida atividade. Como se pode observar, mais da metade, expressa pelo percentual de 55\% tem entre 1 e 5 anos trabalhando com pediatria. Dos sujeitos entrevistados, $15 \%$ tem menos de 1 ano de trabalho, $25 \%$ tem entre 5 e 10 anos. Nenhum indivíduo entrevistado compunha a faixa entre 10 e 15 anos trabalhando na área de enfermagem pediátrica e uma minoria de $5 \%$ referiu trabalhar há mais de 20 anos nesta área da enfermagem. O Quadro 2 é corroborada pelos núme-
$35 \%$ dos profissionais enfermeiros tem menos de 30 anos, seguida das faixas entre 31 e 40 anos e 41 e 50 anos que são compostas cada uma por $30 \%$ da população estudada. Compreendidos na faixa entre 51 e 60 anos, constatou-se apenas 5\% de profissionais atuantes naquela unidade hospitalar, dentre os que foram entrevistados. Acima de 61 anos, não houve índice. 
E12: "Na criança e na família, com preocupação em eficiência técnica sempre. Pois, interfere sempre no bom prognóstico dessa criança e sua família".

E21: "Trabalho com um olhar holístico, focando no paciente e atento aos procedimentos".

Tipo de interação entre enfermeiro e acompanhante da criança hospitalizada horizontal/não hierarquizada ou vertical/hierarquizada

Todos os profissionais têm, pontualmente, seus erros e acertos. Na pesquisa de campo realizada no $\mathrm{HGNI}$, alguns profissionais declararam preferir manter uma relação vertical e hierarquizada com os acompanhantes da criança, o que não parece ser uma postura muito producente. Entretanto, é preciso da parte do entrevistador compreender uma fala honesta, mantendo uma escuta condescendente.

E4: "Uma dose de cada um soma para se chegar a um atendimento individualizado e de qualidade sem exceder. A técnica é importante, mas, a participação do acompanhante também".

E12: "Sempre, minha primeira escolha é horizontal e não hierarquizada. Acredito que o processo de adoecimento e complexo (SIC) e difícil e é necessário trazer conforto e esperança a esse familiar e liberdade para ele se sentir acoIhido".

E18: "Interação horizontal onde a participação é de suma importância para promover uma assistência de qualidade à criança e ao acompanhante".

Especificamente no HGNI, como
Este estudo possibilitou lançar um olhar crítico sobre as relações interpessoais em uma unidade de internação hospitalar no HGNI, no Município de Nova Iguaçu - RJ, uma vez que estas têm repercussão direta sobre as ações destinadas ao cuidado da criança internada. se dá a interação entre o enfermeiro e o acompanhante da criança hospitalizada

O HGNI é um importante hospital para a população de sua região e, deve ser visto como um microcosmo desta realidade. Ali, a forma com que o enfermeiro se relaciona com o paciente infantil e seu acompanhante é crucial para a obtenção de resultados positivos. A grande maioria dos sujeitos entrevistados concorda que no HGNI as relações entre enfermeiros e acompanhantes de crianças hospitalizadas são muito boas e de caráter cooperativo.

E3: "De respeito, informativa, cordial, educada, porém deixando claro seus direitos e deveres diante da realidade que vivemos e todas as dificuldades enfrentadas".

E5: "Não vejo muita paciência e esclarecimento aos familiares (SIC)".

E17: "Não há uma orientação aos acompanhantes. Isso acaba surgindo várias dúvidas aos familiares (SIC), dificultando o trabalho e assistência".

Aspectos Positivos

O principal aspecto positivo que se pode destacar, mediante uma análise da fala dos entrevistados, é que eles possuem uma noção de bom atendimento intrinsecamente relacionada a um cuidar compartilhado entre enfermeiros e acompanhantes na pediatria. Outro aspecto que se pode destacar é que, diante da comunidade, há uma satisfação por parte dos enfermeiros em partilhar conhecimentos, em orientar as pessoas em relação à forma correta de participar dos cuidados, apesar da demanda considerável pelo acesso à instituição. 


\section{Aspectos Negativos}

Como ponto negativo, pode-se destacar que o relacionamento enfermeiro/acompanhante da clínica pediátrica ainda é, de acordo com a fala de alguns entrevistados, vertical e hierarquizado, o que pode gerar insatisfação nos acompanhantes por não se sentirem efetivamente parte integrante do processo de enfrentamento da doença da criança. Estes poucos profissionais precisam mudar esta mentalidade, seja através da sensibilização resultante de uma autocrítica ao próprio trabalho, seja através da educação continuada.

\section{CONCLUSÃO}

Este estudo possibilitou lançar um olhar crítico sobre as relações interpessoais em uma unidade de internação hospitalar no $\mathrm{HGNI}$, no Município de Nova Iguaçu - RJ, uma vez que estas têm repercussão direta sobre as ações destinadas ao cuidado da criança internada.

Pôde-se verificar a importância de estar sempre buscando promover melhorias no processo de comunicação e nas interações que são desenvolvidas, no sentido de considerar o acompanhante como parte fundamental dos cuidados no ambiente hospitalar, tanto no que se refere ao seu direito à orientação para acompanhar e à atenção como usuário.

Verificou-se a importância da equipe de saúde utilizar estratégias de humanização hospitalar no atendimento à família e à criança hospitalizada, considerando suas demandas e promovendo a satisfação daqueles que estão envolvidos.

De acordo com as entrevistas, a equipe de enfermagem do HGNI, num contexto geral, defende a importância da permanência do familiar acompanhante junto à criança hospitalizada, o que favorece o estabelecimento de um clima agradável e contribui na realização dos cuidados e facilita a adesão da criança ao tratamento.

O cuidado da equipe de enfermagem destinado ao familiar/acompanhante relaciona-se à transmissão de orientações educativas. É necessária uma ampliação destas práticas por meio de uma interação que possibilite conhecer os diversos aspectos que são inerentes à vivência da criança, que são de conhecimento da família e que podem influenciar o processo.

Em síntese, este estudo permitiu a discussão de temas comuns vivenciados nas suas práticas, por exemplo, da comunicação enfermeiro/acompanhante na clínica pediátrica e das formas de cuidado, pelo fato de que essas iniciativas contribuem para a qualidade da assistência hospitalar.

\section{Referências}

1. Oliveira TCB, et al. Equipe Multiprofissional de Cuidados Paliativos da Oncologia Pediátrica: Uma Revisão Sistemática. Rev Psicol [Internet]. 2017 [acesso em 14 jun 2018]; 11(35):492-530. Disponível em: http://idonline.emnuvens.com.br/id. 2. Cruz DSM, Pedro SLB, Fassarella CS. Comunicação entre equipe de enfermagem e acompanhante visando à segurança do paciente oncológico durante o processo de hospitalização. Rede de Cuidados em Saúde [Internet]. 2013 [acesso em 26 mar 2018]; 7(1). Disponível em: http:// www.unigranrio.com.br. 3. Facio BC, et al. Internação conjunta pediátrica: compreendendo a negociação enfermeiro-acompanhante. Rev Eletr Enferm [Internet]. 2013 [acesso em 02 mai 2018]; 15(2):447-53. Disponível em: https://www.fen.ufg.br/revista/ v15/n2/pdf/v15n2a18.pdf.

4. Chagas MCS. Criança hospitalizada: cuidado compartilhado entre família e equipe de enfermagem. 2015. 109 f. Dissertação (Mestrado em Enfermagem) - Universidade Federal do Rio Grande, Rio Grande. 2015.

5. Xavier DM, et al. A família revelando-se como um ser de direitos durante a internação hospitalar da criança. Rev Bras Enferm [Internet]. 2013 [acesso em 29 mai 2018]; 66(6):866. Disponível em: http://www.scielo.br/scielo.php?pi$\mathrm{d}=$ S0034-71672013000600009\&script=sci_abstract\&tlng=pt.

6. Azevêdo AVS, et al. Interação equipe de enfermagem, família, e criança hospitalizada: revisão integrativa. Ciência \& Saúde Coletiva [Internet]. 2017 [acesso em 17 abr 2018]; 22:3653-3666. Disponível em: http://www.scielo.br/ scielo.php?pid=S1413-81232017021103653\&script=sci_abstract\&tlng=pt. 7. Macedo IF, et al. As concepções da equipe de enfermagem frente à família da criança hospitalizada. Rev Bras Enferm [Internet]. 2017 [acesso em 19 abr 2018]; 70(5). Disponível em: http://www.scielo.br/scielo.php?script=sci_arttext\&pid=S0034-71672017000500904\&lng=en\&nrm=iso\&tlng=pt.

8. Alcântara KL, et al. Orientações familiares necessárias para uma alta hospitalar segura do recém-nascido prematuro: revisão integrativa. Rev enferm UFPE on line [Internet]. 2017 [acesso em 28 mar 2018]; 11 (2):645-655. Dispo- nível em: https://pdfs.semanticscholar.org/1c26/cb3a22a614c6edb245607ec340ee3fb8a4ed.pdf.

9. Rodrigues FRA, Pereira MLD, Amendoeira J. A transição paradigmática da saúde e suas reflexões na enfermagem como disciplina. Rede de Cuidados em Saúde [Internet]. 2015 [acesso em 14 jun 2018]; 9(1). Disponível em: http://publicacoes.unigranrio.edu.br/index.php/rcs/article/view/2516.

10. Kolhs $M$, et al. Sentimentos de Enfermeiros Frente ao Paciente Oncológico. Journal of Health Sciences [Internet]. 2017 [acesso em 14 mar 2018]; 18(4):245-250. Disponivel em: https://pesquisa.bvsalud.org/portal/resource/ pt/biblio-834028?lang=es.

11. Barros SMM, Andrade MAC, Siqueira FAA. Cuidar de um familiar com câncer: contribuições da terapia familiar sistêmica. Revista Pensando Famílias [Internet]. 2013 [acesso em 21 mai 2018]; 17(2):96-110. Disponível em: http://pepsic.bvsalud.org/scielo.php?script=sci_arttext\&pid=S1679-494X2013000200008.

12. Otani MAP, Barros NF, Marin MJS. A experiência do câncer de mama: percepcões e sentimentos. RBE [Internet]. 2015 [acesso em 27 mar 2018]; 29(3):229. Disponivel em: https://portalseer.ufba.br/index.php/enfermagem/ article/view/12701.

13. Cruz AC, et al. Liga de enfermagem e família e sua interface com a formação em enfermagem pediátrica. Rev. Soc. Bras. Enferm. Ped. [Internet]. 2016 [acesso em 15 jun 2018]; 16(2):75-80. Disponível em: https://sobep.org.br/ revista/images/stories/pdf-revista/vol16-n2/vol_16_n_2-relato_de_experiencia_1.pdf.

14. Bardin L. Análise de Conteúdo. São Paulo: Edições 70; 2011. 15. Marques $C$, et al. 0 cuidador familiar da criança hospitalizada na visão da equipe de enfermagem. Cuidado e Saúde [Internet]. 2014 [acesso em 08 mai 2018]; 13(3). Disponivel em: http://www.periodicos.uem.br/ojs/index.php/ CiencCuidSaude/article/view/22133. 